\title{
Smooth Fit Principle for Impulse Control of Multi-dimensional Diffusion Processes
}

\author{
Xin Guo * Guoliang $\mathrm{Wu}^{\dagger}$
}

February 18, 2008

\begin{abstract}
Value functions of impulse control problems are known to satisfy Quasi-Variational Inequalities (QVI) (Bensoussan and Lions (1982)). This paper proves the smooth-fit $C^{1}$ property of the value function for multi-dimensional controlled diffusions, using a viscosity solution approach. We show by examples how to exploit this regularity property to derive explicitly optimal policy and value function.
\end{abstract}

*Department of Industrial Engineering and Operations Research, University of California at Berkeley, CA 94720-1777. Email address: xinguo@ieor.berkeley.edu

${ }^{\dagger}$ Department of Mathematics, University of California at Berkeley, CA 94720-3840. Email address: guoliang@math . berkeley . edu 


\section{Introduction}

This paper considers the following impulse control problem for an $n$-dimensional diffusion process $X(t)$. In the absence of control, $X(t)$ is governed by an Itô's stochastic differential equation:

$$
d X(t)=\mu(X(t)) d t+\sigma(X(t)) d W(t), \quad X(0)=x,
$$

where $W$ is a standard Brownian motion in a filtered probability space $(\Omega, \mathcal{F}, \mathbb{P})$. If a control policy $V=\left(\tau_{1}, \xi_{1} ; \tau_{2}, \xi_{2} ; \ldots\right)$ is adopted, then $X(t)$ evolves as

$$
d X(t)=\mu(X(t)) d t+\sigma(X(t)) d W(t)+\sum_{i} \delta\left(t-\tau_{i}\right) \xi_{i}
$$

where $\delta(\cdot)$ denotes the Dirac delta function. Here the control $V=\left(\tau_{1}, \xi_{1} ; \tau_{2}, \xi_{2} ; \ldots\right)$ is of an impulse type such that $\tau_{1}, \tau_{2}, \ldots$ is an increasing sequence of stopping times with respect to $\mathcal{F}_{t}$ (the natural filtration generated by $W$ ), and $\xi_{i}$ is an $\mathbb{R}^{n}$-valued, $\mathcal{F}_{\tau_{i}}$-measurable random variable.

The problem is to choose an appropriate impulse control $\left(\tau_{1}, \xi_{1} ; \tau_{2}, \xi_{2} ; \ldots\right)$ so that the following objective function is minimized

$$
\mathbb{E}_{x}\left(\int_{0}^{\infty} e^{-r t} f(X(t)) d t+\sum_{i=1}^{\infty} e^{-r \tau_{i}} B\left(\xi_{i}\right)\right)
$$

Here $f$ is some running cost function, $B$ is the transaction cost, and $r>0$ is a discount factor.

This multi-dimensional control problem has been proposed and studied in various forms in different context of risk management, from optimal cash management [6] to inventory controls [14, 13, 34, 33]. More recent papers in the literature of mathematical finance include those on transaction cost in portfolio management [2, 19, 20, 9, 25, 28, insurance models [16, 4], liquidity risk [22, 3], optimal control of exchange rates [17, 26, 5], and finally real options [35, 23].

Compared to regular controls, impulse control provides a more natural mathematical framework when the state space is discontinuous. It is a more general version of singular control allowing for non-zero fixed cost [13] and therefore harder to analyze. Indeed, in contrast to the singular/regular control theory, which enjoys a vast literature in financial engineering (see for instance Merton [24] and Karatzas and Shreve [18] among others), impulse control is less well-understood, especially in terms of regularity properties of the value function and the structure of the optimal policy. In fact, regarding optimal policy, the best known work is perhaps still due to [6], which characterized the $(u, U, d, D)$ form of the optimal policy for an inventory system. Although there have been various extensions of this structural result [14, 13, 34, 33, 30], most were derived through the verification theorem approach and by assuming a priori the smooth-fit property through the action/continuation regions. In the end, this approach usually amounts to solving complex algebraic equations that are hard to verify without a priori knowledge of the regularity property, thus the correctness 
of the "solution" is dubious. In [1] the value functions were shown to be the solutions of Quasi-Variational-Inequalities and the regularity properties were established when the control is strictly positive and the state space is in a bounded region. However, to the best of our knowledge, regularity properties for value functions involving all-direction controls have not been fully established. This is an important omission in light of the wide range of applications mentioned earlier. (Special cases of one-dimensional singular control and closely related switching control problems were studied recently respectively by [12] and [31.)

Our Work. This paper studies regularity properties of impulse control problem (1.3) on multi-dimensional diffusions. Unlike the approach in [1] where the regularity was established through studying the corresponding QVI, we first prove the value function to be the unique viscosity solution to the corresponding HJB equation. The main difficulty in proving the uniqueness of the viscosity solution is the unusual non-local property of the associated operator in the HJB equation and the unboundedness of the state space. We overcome this by exploiting and clarifying the definition of viscosity solutions in a local sense and by relating the problem to an optimal stopping problem (see also Remark 1). Next, we establish the regularity property of the value function, and in particular, the smooth-fit $C^{1}$ property through the boundaries between action and continuation regions. The existing technique in [1] does not apply here as it relies on certain smoothness assumption that fails in our case (see also Remark 2). Finally, we show how to exploit this smooth-fit property to explicitly derive the form of optimal policy and the action/continuation regions for special cases that were first studied and analyzed in [6].

\section{Formulation and Assumptions}

\subsection{Model Formulation}

Let us first define precisely the family of admissible controls. An admissible impulse control $V$ consists of a sequence of stopping times $\tau_{1}, \tau_{2}, \ldots$ with respect to $\mathcal{F}_{t}$ (the natural filtration generated by $W$ ) and a corresponding sequence of $\mathbb{R}^{n}$-valued random variables $\xi_{1}, \xi_{2}, \ldots$ satisfying the conditions

$$
\left\{\begin{array}{l}
0 \leq \tau_{1} \leq \tau_{2} \leq \cdots \leq \tau_{i} \leq \ldots \\
\tau_{i} \rightarrow \infty \text { a.s. as } i \rightarrow \infty \\
\xi_{i} \in \mathcal{F}_{\tau_{i}}, \quad \forall i \geq 1
\end{array}\right.
$$

As explained in the introduction, given an initial state $x \in \mathbb{R}^{n}$ and an admissible control $V=\left(\tau_{1}, \xi_{1} ; \tau_{2}, \xi_{2} ; \ldots\right)$, the underlying process $X(t)$ is governed by the stochastic differential equation

$$
\left\{\begin{array}{l}
d X(t)=\mu(X(t)) d t+\sigma(X(t)) d W(t)+\sum_{i} \delta\left(t-\tau_{i}\right) \xi_{i} \\
X(0)=x
\end{array}\right.
$$


where $\delta(\cdot)$ denotes the Dirac delta function. The associated total expected cost (objective function) is given by

$$
J_{x}[V]:=\mathbb{E}_{x}\left(\int_{0}^{\infty} e^{-r t} f(X(t)) d t+\sum_{i=1}^{\infty} e^{-r \tau_{i}} B\left(\xi_{i}\right)\right),
$$

where $f$ is the "running cost", $B$ is the "transaction cost" and $r>0$ is the discount factor. We will specify the conditions on $f$ and $B$ in $\$ 2.2$ below.

The goal is to find an admissible $\widetilde{V}$ to minimize the total cost, i.e.,

$$
J_{x}[\tilde{V}] \leq J_{x}[V], \quad \text { for any admissible } V .
$$

We define the value function

$$
u(x)=\inf _{V} J_{x}[V]
$$

where the infimum is taken over all admissible control policies.

\subsection{Assumptions and Notations}

Throughout this paper, we shall impose the following standing assumptions:

(A1) Lipschitz conditions on $\mu, \sigma: \mathbb{R}^{n} \rightarrow \mathbb{R}$ : there exist constants $\operatorname{Lip}_{\mu}$, $\operatorname{Lip}_{\sigma}>0$ such that

$$
\left\{\begin{array}{l}
|\mu(x)-\mu(y)| \leq \operatorname{Lip}_{\mu}|x-y|, \\
|\sigma(x)-\sigma(y)| \leq \operatorname{Lip}_{\sigma}|x-y|,
\end{array} \quad \forall x, y \in \mathbb{R}^{n} .\right.
$$

(A2) Lipschitz condition on the running cost $f \geq 0$ : there exist constants $\operatorname{Lip}_{f}>0$ such that

$$
|f(x)-f(y)| \leq \operatorname{Lip}_{f}|x-y|, \quad \forall x, y \in \mathbb{R}^{n} .
$$

(A3) Conditions on the transaction cost function $B: \mathbb{R}^{n} \rightarrow \mathbb{R}$ :

$$
\left\{\begin{array}{l}
\inf _{\xi \in \mathbb{R}^{n}} B(\xi)=K>0, \\
B \in C\left(\mathbb{R}^{n} \backslash\{0\}\right), \\
|B(\xi)| \rightarrow \infty, \text { as }|\xi| \rightarrow \infty, \text { and } \\
B\left(\xi_{1}\right)+B\left(\xi_{2}\right) \geq B\left(\xi_{1}+\xi_{2}\right)+K, \quad \forall \xi_{1}, \xi_{2} \in \mathbb{R}^{n} .
\end{array}\right.
$$

(A4) $r>2 \operatorname{Lip}_{\mu}+\operatorname{Lip}_{\sigma}^{2}$. 
We will also use the following notations for the operators

$$
\begin{aligned}
& \mathcal{M} \varphi(x)=\inf _{\xi \in \mathbb{R}^{n}}(\varphi(x+\xi)+B(\xi)), \\
& \mathcal{L} \varphi(x)=-\operatorname{tr}\left[A \cdot D^{2} \varphi(x)\right]-\mu(x) \cdot D \varphi(x)+r \varphi(x),
\end{aligned}
$$

where the matrix $A=\left(a_{i j}\right)_{n \times n}=\frac{1}{2} \sigma(x) \sigma(x)^{\top}$.

Denote by $\Xi(x)$ the set of all the points $\xi$ for which $\mathcal{M} u$ achieve the minimum, i.e.,

$$
\Xi(x):=\left\{\xi \in \mathbb{R}^{n}: \mathcal{M} u(x)=u(x+\xi)+B(\xi)\right\} .
$$

We also adopt the following standard notations for function spaces:

$$
\begin{aligned}
U C\left(\mathbb{R}^{n}\right)= & \text { space of all uniformly continuous functions on } \mathbb{R}^{n}, \\
U C_{b b}\left(\mathbb{R}^{n}\right)= & \left\{f \in U C\left(\mathbb{R}^{n}\right): f \text { is bounded below }\right\}, \\
W^{k, p}(U)= & \text { space of all } L^{p} \text { functions with } \beta \text {-th weak partial } \\
& \text { derivatives belonging to } L^{p}, \forall|\beta| \leq k, \\
C_{c}^{\infty}(U)= & \left\{f \in C^{\infty}(U): f \text { has compact support in } U\right\}, \\
C^{k, \alpha}(D)= & \left\{f \in C^{k}(D): \sup _{x, y \in D}\left\{\frac{\left|D^{\beta} f(x)-D^{\beta} f(y)\right|}{|x-y|^{\alpha}}\right\}<\infty, \forall|\beta| \leq k\right\} .
\end{aligned}
$$

\subsection{Preliminary Results}

We first establish some preliminary results about the value function as well as the operator $\mathcal{M}$, under the standing assumptions.

Lemma 2.1. The value function $u(x)$ defined by $(2.3)$ is Lipschitz.

Proof. Given an admissible control $V=\left(\tau_{1}, \xi_{1} ; \tau_{2}, \xi_{2} ; \ldots\right)$, and two initial states $x_{1}, x_{2}$, denote by $x^{i}(t)$ the solution of (2.1) with initial state $x_{i}(i=1,2)$. Applying Itô's formula to $Y(t)=\left|x^{1}(t)-x^{2}(t)\right|^{2}$ and using (2.4), we obtain,

$$
\begin{aligned}
& \mathbb{E} Y(t) \leq C_{1} \int_{0}^{t} \mathbb{E} Y(s) d s+\left|x_{1}-x_{2}\right|^{2}, \\
\Rightarrow & \mathbb{E}\left|x^{1}(t)-x^{2}(t)\right| \leq e^{C_{1} t}\left|x_{1}-x_{2}\right| \text { (Gronwall's Inequality), }
\end{aligned}
$$

where $C_{1}=2 \operatorname{Lip}_{\mu}+\operatorname{Lip}_{\sigma}^{2}$. By Lipschitz condition on $f$,

$$
\begin{aligned}
J_{x_{1}}[V]-J_{x_{2}}[V] & =\mathbb{E}\left(\int_{0}^{\infty} e^{-r t}\left(f\left(x^{1}(t)\right)-f\left(x^{2}(t)\right)\right) d t\right) \\
& \leq C\left(\int_{0}^{\infty} e^{-r t} e^{C_{1} t}\left|x_{1}-x_{2}\right| d t\right) \\
& =C\left|x_{1}-x_{2}\right| .
\end{aligned}
$$


Since $V$ is arbitrary,

$$
u\left(x_{1}\right) \leq J_{x_{1}}[V] \leq J_{x_{2}}[V]+C\left|x_{1}-x_{2}\right| \Rightarrow u\left(x_{1}\right) \leq u\left(x_{2}\right)+C\left|x_{1}-x_{2}\right| .
$$

Exchanging the roles of $x_{1}, x_{2}$ we get the desired result.

Lemma 2.2 (Basic properties of $\mathcal{M}$ ).

1. $\mathcal{M}$ is concave: for any $\varphi_{1}, \varphi_{2} \in C\left(\mathbb{R}^{n}\right)$ and $0 \leq \lambda \leq 1$,

$$
\mathcal{M}\left(\lambda \varphi_{1}+(1-\lambda) \varphi_{2}\right) \geq \lambda \mathcal{M} \varphi_{1}+(1-\lambda) \mathcal{M} \varphi_{2} .
$$

2. $\mathcal{M}$ is increasing: for any $\varphi_{1} \leq \varphi_{2}$ everywhere,

$$
\mathcal{M} \varphi_{1} \leq \mathcal{M} \varphi_{2} .
$$

3. The operator $\mathcal{M}$ maps $C\left(\mathbb{R}^{n}\right)$ into $C\left(\mathbb{R}^{n}\right)$. In particular, $\mathcal{M} u(\cdot)$ is continuous. Moreover, $\mathcal{M}$ maps $U C\left(\mathbb{R}^{n}\right)$ into $U C\left(\mathbb{R}^{n}\right)$ and maps a Lipschitz function to a Lipschitz function.

Proof. (1) and (2) are obvious.

(3) Suppose $\varphi \in C\left(\mathbb{R}^{n}\right)$. Then for any $x \in \mathbb{R}^{n}, \xi \in \mathbb{R}^{n}, \varepsilon>0$,

$$
-\varepsilon<\varphi(x+\xi+y)-\varphi(x+\xi)<\varepsilon,
$$

provided that $|y|<\delta$ sufficiently small. Hence

$$
\varphi(x+\xi)+B(\xi)-\varepsilon<\varphi(x+\xi+y)+B(\xi)<\varphi(x+\xi)+B(\xi)+\varepsilon .
$$

This holds for arbitrary $\xi$, so by taking infimum we get

$$
\mathcal{M} \varphi(x)-\varepsilon \leq \mathcal{M} \varphi(x+y) \leq \mathcal{M} \varphi(x)+\varepsilon,
$$

provided $|y|<\delta$ is small enough.

The last statement can be proved similarly.

Lemma 2.3. $u$ and $\mathcal{M} u$ defined as above satisfy $u(x) \leq \mathcal{M} u(x)$ for all $x \in \mathbb{R}^{n}$.

Proof. Suppose $x \in \mathbb{R}^{n}, \xi \in \mathbb{R}^{n}$, and $V=\left(\tau_{1}, \xi_{1} ; \tau_{2}, \xi_{2} ; \ldots\right)$ is an admissible control policy. Then $V^{\prime}=\left(0, \xi ; \tau_{1}, \xi_{1} ; \tau_{2}, \xi_{2} ; \ldots\right)$ is also admissible. Moreover,

$$
u(x) \leq J_{x}\left[V^{\prime}\right]=J_{x+\xi}[V]+B(\xi) .
$$

Taking the infimum over $V$ and then infimum over $\xi \in \mathbb{R}^{n}$, we get $u(x) \leq \mathcal{M} u(x)$.

Now define the continuation region $\mathcal{C}$ and the action region $\mathcal{A}$ as follows,

$$
\begin{aligned}
\mathcal{C} & :=\left\{x \in \mathbb{R}^{n}: u(x)<\mathcal{M} u(x)\right\}, \\
\mathcal{A} & :=\left\{x \in \mathbb{R}^{n}: u(x)=\mathcal{M} u(x)\right\} .
\end{aligned}
$$

Then, since $u$ and $\mathcal{M} u$ are continuous, we have 
Proposition 1. $\mathcal{C}$ is open.

Furthermore,

Proposition 2. Suppose $x \in \mathcal{A}$, then

(1) The set

$$
\Xi(x):=\left\{\xi \in \mathbb{R}^{n}: \mathcal{M} u(x)=u(x+\xi)+B(\xi)\right\}
$$

is nonempty, i.e., the infimum is in fact a minimum.

(2) Moreover, for any $\xi(x) \in \Xi(x)$, we have

$$
u(x+\xi(x)) \leq \mathcal{M} u(x+\xi(x))-K,
$$

in particular,

$$
x+\xi(x) \in \mathcal{C} .
$$

Proof. (1) Given $x \in \mathcal{A}$, take sequence $\left\{\xi_{n}\right\}$ such that

$$
\mathcal{M} u(x) \leq u\left(x+\xi_{n}\right)+B\left(\xi_{n}\right) \leq \mathcal{M} u(x)+\frac{1}{n} .
$$

Then $\left\{\xi_{n}\right\}$ is bounded since $|B(\xi)| \rightarrow \infty$ as $|\xi| \rightarrow \infty$. Extract a convergent subsequence $\left\{\xi_{n_{k}}\right\}$ that converges to $\xi^{*}$.

Claim: $\xi^{*} \neq 0$ and $\mathcal{M} u(x)=u\left(x+\xi^{*}\right)+B\left(\xi^{*}\right)$. Suppose $\xi^{*}=0$, since

$$
u\left(x+\xi_{n_{k}}\right)+K \leq u\left(x+\xi_{n_{k}}\right)+B\left(\xi_{n_{k}}\right) \leq \mathcal{M} u(x)+\frac{1}{n_{k}},
$$

sending $k \rightarrow \infty$, we deduce that $u(x)+K \leq \mathcal{M} u(x)=u(x)$. Contradiction. Now that $\xi^{*} \neq 0$, clearly $\xi^{*} \in \Xi(x)$ since $B(\cdot)$ is also continuous at $\xi^{*} \neq 0$.

(2) Recall that $B\left(\xi_{1}\right)+B\left(\xi_{2}\right) \geq K+B\left(\xi_{1}+\xi_{2}\right)$, hence

$$
\begin{aligned}
\mathcal{M} u(x) & =\inf _{\xi \in \mathbb{R}}(u(x+\xi)+B(\xi)) \\
& =\inf _{\eta \in \mathbb{R}}(u(x+\xi(x)+\eta)+B(\xi(x)+\eta) \\
& \leq \inf _{\eta \in \mathbb{R}}[u(x+\xi(x)+\eta)+B(\eta)]+B(\xi(x))-K \\
& =\mathcal{M} u(x+\xi(x))+B(\xi(x))-K .
\end{aligned}
$$

On the other hand, $\mathcal{M} u(x)=u(x+\xi(x))+B(\xi(x))$. We get the desired result.

\section{Value Function as Viscosity Solution}

We show in this section that under certain conditions, the value function of the impulse control problem is the unique viscosity solution of the corresponding Hamilton-Jacobi-Bellman equation

$$
\max (\mathcal{L} u-f, u-\mathcal{M} u)=0 .
$$




\subsection{Definition of Viscosity Solutions}

First, recall [21] the following definition of viscosity subsolutions (supersolutions, resp.):

(a) If $\varphi \in C^{2}\left(\mathbb{R}^{n}\right), u-\varphi$ has a global maximum (minimum, resp.) at $x_{0}$ and $u\left(x_{0}\right)=\varphi\left(x_{0}\right)$, then

$$
\max \left(\mathcal{L} \varphi\left(x_{0}\right)-f\left(x_{0}\right), \varphi\left(x_{0}\right)-\mathcal{M} \varphi\left(x_{0}\right)\right) \leq 0 \quad(\geq 0 \text { resp. })
$$

However, note that the operator $\mathcal{M}$ is non-local, i.e., $\mathcal{M} \varphi\left(x_{0}\right)$ is not determined by values of $\varphi$ in a neighborhood of $x_{0}$, and $\mathcal{M} \varphi\left(x_{0}\right)$ might be very small if $\varphi$ is small away from $x_{0}$. Therefore, one has no control over $\mathcal{M} \varphi\left(x_{0}\right)$ by simply requiring that $u-\varphi$ has a local maximum (minimum) at $x_{0}$. In light of this, one can modify the definition of viscosity subsolutions (supersolutions, resp.) as follows: Suppose $u \in U C\left(\mathbb{R}^{n}\right)$,

(b) If $\varphi \in C^{2}\left(\mathbb{R}^{n}\right), u-\varphi$ has a local maximum (minimum, resp.) at $x_{0}$ and $u\left(x_{0}\right)=\varphi\left(x_{0}\right)$, then

$$
\max \left(\mathcal{L} \varphi\left(x_{0}\right)-f\left(x_{0}\right), u\left(x_{0}\right)-\mathcal{M} u\left(x_{0}\right)\right) \leq 0 \quad(\geq 0 \text { resp. })
$$

In fact, one can show that

Theorem 3.1. The above two definitions of viscosity subsolutions (supersolutions, resp.) are equivalent.

Proof. We will only prove the equivalence of subsolutions.

"(b) $\Rightarrow(\mathrm{a}) "$. Suppose $\varphi \in C^{2}\left(\mathbb{R}^{n}\right), u-\varphi$ has a global maximum at $x_{0}$ and $u\left(x_{0}\right)=\varphi\left(x_{0}\right)$, then $u \leq \varphi$ globally and by Lemma 2.2 ,

$$
\varphi\left(x_{0}\right)=u\left(x_{0}\right) \leq \mathcal{M} u\left(x_{0}\right) \leq \mathcal{M} \varphi\left(x_{0}\right) .
$$

"(a) $\Rightarrow(\mathrm{b}) "$. Suppose $\varphi \in C^{2}\left(\mathbb{R}^{n}\right), u-\varphi$ has a local maximum at $x_{0}$ and $u\left(x_{0}\right)=\varphi\left(x_{0}\right)$. For any $\varepsilon>0$, take $r>0$ so small that

$$
u \leq \varphi \leq u+\varepsilon \text { in } \bar{B}_{2 r}\left(x_{0}\right):=\left\{x \in \mathbb{R}^{n}:\left|x-x_{0}\right| \leq 2 r\right\}
$$

There also exists a function $\tilde{\varphi} \in C^{\infty}\left(\mathbb{R}^{n}\right)$ such that

$$
u \leq \tilde{\varphi} \leq u+\varepsilon \text { in } \mathbb{R}^{n} .
$$

(For instance, the usual mollification $\tilde{\varphi}=u * \eta^{\delta}+\varepsilon$, with $\delta>0$ small enough.) Take a cutoff function $\zeta(x)$ such that

$$
0 \leq \zeta(x) \leq 1 ; \zeta \equiv 1 \text { on } \bar{B}_{r}\left(x_{0}\right) ; \zeta \equiv 0 \text { off } \bar{B}_{2 r}\left(x_{0}\right)
$$

Now define

$$
\psi(x)=\zeta(x) \varphi(x)+(1-\zeta(x)) \tilde{\varphi}(x) .
$$


Then clearly by construction,

$$
u(x) \leq \psi(x) \leq u(x)+\varepsilon,
$$

and $\psi$ attains a global maximum at $x_{0}$. Thus

$$
\mathcal{L} \psi\left(x_{0}\right)-f\left(x_{0}\right) \leq 0 ; \quad \psi\left(x_{0}\right) \leq \mathcal{M} \psi\left(x_{0}\right)
$$

Note that $\psi\left(x_{0}\right)=\varphi\left(x_{0}\right)=u\left(x_{0}\right), D \psi\left(x_{0}\right)=D \varphi\left(x_{0}\right), D^{2} \psi\left(x_{0}\right)=D^{2} \varphi\left(x_{0}\right)$, we have

$$
\mathcal{L} \varphi\left(x_{0}\right)-f\left(x_{0}\right) \leq 0 ; \quad u\left(x_{0}\right) \leq \mathcal{M} \psi\left(x_{0}\right) \leq \mathcal{M} u\left(x_{0}\right)+\varepsilon .
$$

Finally, since $\varepsilon>0$ is arbitrary, sending it to 0 , we have (3.2).

In light of Theorem 3.1, throughout the paper we shall adopt the following definition of viscosity solution:

Definition 1. The function $u$ is called a viscosity solution of $(\overline{\mathrm{HJB}})$, if

(1) (Subsolution Property) For any $\varphi \in C^{2}\left(\mathbb{R}^{n}\right), u-\varphi$ has a local maximum at $x_{0}$ and $u\left(x_{0}\right)=\varphi\left(x_{0}\right)$, we have

$$
\max \left(\mathcal{L} \varphi\left(x_{0}\right)-f\left(x_{0}\right), u\left(x_{0}\right)-\mathcal{M} u\left(x_{0}\right)\right) \leq 0
$$

(2) (Supersolution Property) For any $\varphi \in C^{2}\left(\mathbb{R}^{n}\right), u-\varphi$ has a local minimum at $x_{0}$ and $u\left(x_{0}\right)=\varphi\left(x_{0}\right)$, we have

$$
\max \left(\mathcal{L} \varphi\left(x_{0}\right)-f\left(x_{0}\right), u\left(x_{0}\right)-\mathcal{M} u\left(x_{0}\right)\right) \geq 0 .
$$

Then we have the following known result [29]. (For readers' convenience, we provide the proof in Appendix A).

Theorem 3.2. The value function defined by $(2.3)$ is a viscosity solution of the HamiltonJacobi-Bellman equation

$$
\max \{\mathcal{L} u-f, u-\mathcal{M} u\}=0
$$

\subsection{Uniqueness of Viscosity Solution}

In this section we shall show that the viscosity solution for Eq. (HJB) is unique in $U C_{b b}\left(\mathbb{R}^{n}\right)$.

The key idea is to relate the impulse control problem to an optimal stopping problem via the following operator $\mathcal{T}$, as in Bensoussan-Lions [1] and Ramaswamy-Dharmatti [32]. More precisely, given $u \in U C\left(\mathbb{R}^{n}\right)$, consider the following optimal stopping time problem

$$
\mathcal{T} u(x)=\inf _{\tau} \mathbb{E}\left(\int_{0}^{\tau} e^{-r t} f(X(t)) d t+e^{-r \tau} \mathcal{M} u(x(\tau))\right)
$$

subject to Eq. (1.1) and with the infimum taken over all $\mathcal{F}_{t}$ stopping times.

We shall first prove the uniqueness of viscosity solution to the Hamilton-Jacobi-Bellman equation (3.7) associated with this optimal stopping problem (3.5). We then exploit the property of the operator $\mathcal{T}$ to establish the uniqueness of the viscosity solution to (HJB) for the impulse control problem. 


\subsubsection{Related Optimal Stopping Problems}

Now given Eq. (1.1), consider the following more generic optimal stopping problem with a terminal cost $g(\cdot)$ :

$$
v(x)=\inf _{\tau} \mathbb{E}\left(\int_{0}^{\tau} e^{-r t} f(X(t)) d t+e^{-r \tau} g(x(\tau))\right)
$$

where $f$ is the same as before, and the infimum is taken over all $\mathcal{F}_{t}$ stopping times.

First, the following result is well-known [27].

Proposition 3. Assume that $g \in C\left(\mathbb{R}^{n}\right)$. Then the value function $v(x)$ defined by (3.6) is a continuous viscosity solution of the Hamilton-Jacobi-Bellman equation

$$
\max \{\mathcal{L} w-f, w-g\}=0, \text { in } \mathbb{R}^{n}
$$

where $\mathcal{L}$ is defined in (2.8).

Next, we show

Theorem 3.3. [Unique Viscosity Solution for Optimal Stopping] Suppose $g \in U C\left(\mathbb{R}^{n}\right)$ and suppose there are some constants $C, \Lambda>0$, such that

$$
\begin{cases}|\mu(x)| \leq C, & \forall x \in \mathbb{R}^{n}, \\ a_{i j}(x) \xi_{i} \xi_{j} \leq \Lambda|\xi|^{2}, & \forall x, \xi \in \mathbb{R}^{n},\end{cases}
$$

where $\left(a_{i j}(x)\right)_{n \times n}=\frac{1}{2} \sigma(x) \sigma(x)^{\top}$. Then (3.7) has only one viscosity solution in $U C\left(\mathbb{R}^{n}\right)$.

To prove Theorem 3.3 , the following observation is useful.

Lemma 3.4. $w$ is a viscosity solution of $\max \{\mathcal{L} w-f, w-g\}=0$ if and only if it is a viscosity solution of

$$
F\left(x, w(x), D w(x), D^{2} w(x)\right)=0,1 \text {. }
$$

where $F: \mathbb{R}^{n} \times \mathbb{R} \times \mathbb{R}^{n} \times S^{n} \rightarrow \mathbb{R}$ is defined by

$$
\begin{aligned}
& F(x, t, p, X)=r t+\max \{G(x, p, X),-r g(x)\}, \\
& G(x, p, X)=-\operatorname{tr}[A X]-\mu(x) \cdot p-f(x) .
\end{aligned}
$$

The key to proving Theorem 3.3 is the following comparison result.

Lemma 3.5. Let $D \subset \mathbb{R}^{n}$ be a bounded open set, $f, g \in U C\left(\mathbb{R}^{n}\right)$, and $u \in C(\bar{D})$ be a subsolution and $v \in C(\bar{D})$ a supersolution of

$$
\max \{\mathcal{L} w-f, w-g\}=0 \text {, in } D .
$$

Assume also that $u \leq v$ on $\partial D$. Then $u \leq v$ in $\bar{D}$.

\footnotetext{
${ }^{1}$ When there is no risk of confusion, we will also abbreviate $F=0$ for equation (3.9)
} 
The proof of lemma 3.5 is based on the classical comparison theorem of second order degenerate elliptic differential equations in bounded domains (Cf. [7]), and we defer it to Appendix B.

The following Lemma (see Theorem 1 and the remarks on the fully nonlinear case in [8]) extends the above comparison result from bounded domains to an unbounded one.

Lemma 3.6. Suppose $F(x, t, p, X)$ is elliptic in $X$ such that there exist constants $\alpha, C, \Lambda>0$ satisfying

$$
F(x, t+s, p+q, X+Y) \geq F(x, t, p, X)+\alpha s-C|q|-\Lambda \operatorname{tr}(Y),
$$

for all $x, p, q \in \mathbb{R}^{n}, t \in \mathbb{R}, s \geq 0, X, Y \in S^{n}, Y \geq 0$. Suppose also that we have comparison result for the equation $F=0$ in bounded domains. If $u$ and $v$ are continuous sub-and supersolution of $F\left(x, u(x), D u(x), D^{2} u(x)\right)=0$ in $\mathbb{R}^{n}$ with at most polynomial growth, then

$$
u \leq v \text { in } \mathbb{R}^{n}
$$

Now we can return to

Proof of Theorem 3.3. By Lemma 3.5 and Lemma 3.6, and noticing also

$$
w \in U C\left(\mathbb{R}^{n}\right) \Rightarrow \sup _{x \in \mathbb{R}^{n}} \frac{|w(x)|}{1+|x|}<\infty
$$

it remains to show that $F$, defined by (3.10), satisfies (3.12).

$$
\begin{aligned}
& F(x, t+s, p+q, X+Y)-F(x, t, p, X) \\
= & r s+\max \{G(x, p+q, X+Y),-r g(x)\}-\max \{G(x, p, X),-r g(x)\} \\
\geq & \left\{\begin{array}{l}
r s, \text { if } G(x, p, X) \leq-r g(x), \\
r s+G(x, p+q, X+Y)-G(x, p, X), \text { otherwise. }
\end{array}\right.
\end{aligned}
$$

If $Y \geq 0$, using (3.8), we have

$$
\begin{aligned}
G(x, p+q, X+Y)-G(x, p, X) & =-\operatorname{tr}(A Y)+\mu(x) q \\
& \geq-C|q|-\Lambda \operatorname{tr}(Y),
\end{aligned}
$$

since $\operatorname{tr}(A Y)=\operatorname{tr}\left(S^{\top} A S\right) \leq C \operatorname{tr}\left(S^{\top} S\right)=\Lambda \operatorname{tr}(Y)$, where $A=\frac{1}{2} \sigma \sigma^{\top}, Y=S S^{\top}$. This completes the proof.

\subsubsection{Uniqueness for Impulse Control Problems}

Now we are ready to prove 
Theorem 3.7 (Unique Viscosity Solution for Impulse Controls). Assume that there are some constants $C, \Lambda>0$, such that

$$
\begin{cases}|\mu(x)| \leq C, & \forall x \in \mathbb{R}^{n}, \\ a_{i j}(x) \xi_{i} \xi_{j} \leq \Lambda|\xi|^{2}, & \forall x, \xi \in \mathbb{R}^{n},\end{cases}
$$

and $r>\operatorname{Lip}_{f}$. Then the value function for the impulse control problem defined by (2.3) is a unique viscosity solution in $U C_{b b}\left(\mathbb{R}^{n}\right)$ for $E q$. (HJB).

The proof relies on the following properties of the operator $\mathcal{T}$.

\section{Lemma 3.8.}

1. $\mathcal{T}: U C\left(\mathbb{R}^{n}\right) \rightarrow U C\left(\mathbb{R}^{n}\right) ;$

2. If $u \leq v$ in $\mathbb{R}^{n}$, then $\mathcal{T} u \leq \mathcal{T} v$ in $\mathbb{R}^{n}$;

3. $\mathcal{T}$ is concave on $U C\left(\mathbb{R}^{n}\right)$.

Lemma 3.8 is immediate by the monotone and concave properties of $\mathcal{M}$ in Lemma 2.2 , and by a direct application of Itô's formula and Gronwall's inequality (Cf. Lemma 2.1).

Proof of Theorem 3.7. Suppose $u, v \in U C\left(\mathbb{R}^{n}\right)$ are two solutions of (HJB). Without loss of generality, we can assume $u, v \geq 0$. Otherwise if $-C$ is a lower bound then $u+C, v+C$ are nonnegative solutions to Eq. (HJB) of the same structure with $f$ replaced by $f+r C$.

First, by definition of $\mathcal{T}$ together with Proposition 3 and Theorem $3.3, \mathcal{T} u$ is the unique viscosity solution of

$$
\max \{\mathcal{L}(\mathcal{T} u)-f, \mathcal{T} u-\mathcal{M} u\}=0, \text { in } \mathbb{R}^{n} .
$$

On the other hand, $u$ is also a viscosity solution of

$$
\max \{\mathcal{L} u-f, u-\mathcal{M} u\}=0, \text { in } \mathbb{R}^{n} .
$$

By uniqueness, $u=\mathcal{T} u$. Similarly, $v=\mathcal{T} v$.

Next, it suffices to show that if $u, v$ satisfies $u-v \leq \gamma u$ for some $\gamma \in[0,1]$, then there exists some constant $\nu \in(0,1)$ such that

$$
u-v \leq \gamma(1-\nu) u
$$

Indeed, if this claim holds, then note that $u-v \leq u$ since $v \geq 0$, we have $u-v \leq(1-\nu) u$. Repeating the argument, we have

$$
u-v \leq(1-\nu)^{n} u
$$

Sending $n \rightarrow \infty$, we get $u \leq v$. Switching the roles of $u$ and $v$, we get $u=v$ as desired.

Now it remains to check claim (3.13). First, by concavity of $\mathcal{T}$, we have

$$
\mathcal{T} v \geq \mathcal{T}[(1-\gamma) u] \geq(1-\gamma) \mathcal{T} u+\gamma \mathcal{T} 0
$$


Since $f$ is at most with a linear growth,

$$
\begin{aligned}
u & =\mathcal{T} u=\inf _{\tau} \mathbb{E}\left(\int_{0}^{\tau} e^{-r t} f(X(t)) d t+e^{-r \tau} \mathcal{M} u(x(\tau))\right) \\
& \leq \mathbb{E} \int_{0}^{\infty} e^{-r t} f(X(t)) d t \leq C \int_{0}^{\infty} e^{-r t}(1+\mathbb{E}|X(t)|) d t \\
& \leq C(1+|x|) \int_{0}^{\infty} e^{-r t} e^{C t} d t=: u_{0}<\infty .
\end{aligned}
$$

Here, the last line follows from the Gronwall's inequality.

Thus, $\mathcal{M} u(x)=\inf (u(x+\xi)+B(\xi)) \leq u_{0}+K=K / \nu$, where

$$
\nu:=\frac{K}{K+u_{0}} \in(0,1) \text {. }
$$

Note that $\mathcal{M} 0=K$, we obtain

$$
\begin{aligned}
\mathcal{T} 0 & =\inf _{\tau} \mathbb{E}\left(\int_{0}^{\tau} e^{-r t} f(X(t)) d t+e^{-r \tau} K\right) \\
& \geq \nu \inf _{\tau} \mathbb{E}\left(\int_{0}^{\tau} e^{-r t} f(X(t)) d t+e^{-r \tau} \mathcal{M} u(X(t))\right)=\nu \mathcal{T} u .
\end{aligned}
$$

Therefore,

$$
\mathcal{T} v \geq(1-\gamma) \mathcal{T} u+\gamma \mathcal{T} 0 \geq(1-\gamma) \mathcal{T} u+\gamma \nu \mathcal{T} u
$$

The claim (3.13) is now clear by plugging in $\mathcal{T} u=u, \mathcal{T} v=v$.

Remark 1. It is worth noting that in [3] uniqueness of viscosity solution was characterized for the value function of a finite horizon impulse control problem with execution delay involving the liquidity risk. However, their technique relies on the particular setup of a positive delay parameter and can not be reduced to our case.

\section{Regularity of Value Function}

We shall show in this section that the value function $u$ is in the Sobolev space $W^{2, p}(\mathcal{O})$ for any open bounded region $\mathcal{O}$, and in particular, $u \in C^{1}\left(\mathbb{R}^{n}\right)$.

Recall

$$
\begin{aligned}
& \mathcal{C}:=\left\{x \in \mathbb{R}^{n}: u(x)<\mathcal{M} u(x)\right\} \\
& \mathcal{A}:=\left\{x \in \mathbb{R}^{n}: u(x)=\mathcal{M} u(x)\right\} .
\end{aligned}
$$

First, $u(\cdot)$ is clearly $C^{2}$ in the continuation region $\mathcal{C}$. 
Lemma $4.1\left(C^{2}\right.$-Regularity in $\left.\mathcal{C}\right)$. (1) The value function $u(\cdot) \in C^{2}(\mathcal{C})$, and satisfies the following differential equation in the classical sense,

$$
\mathcal{L} u(x)-f(x)=0, \quad x \in \mathcal{C} .
$$

(2) For any set $D \Subset \mathcal{C}^{2}$,

$$
u \in C^{2, \alpha}(\bar{D})
$$

for some $\alpha>0$.

Proof. Recall that from Theorem 3.2, $u$ satisfies (4.1) in $\mathcal{C}$ in a viscosity sense.

Now for any open ball $B \subset \mathcal{C}$, consider the Dirichlet problem

$$
\begin{cases}\mathcal{L} w-f=0, & \text { in } B, \\ w=u, & \text { on } \partial B .\end{cases}
$$

Classical Schauder estimates (Cf. [11, Theorem 6.13]) ensures that such a solution $w$ exists and belongs to $C^{2, \alpha}(B)$ because $f \in C^{0, \alpha}(B)$ for some $\alpha>0$. Thus $w$ satisfies the differential equation in viscosity sense, whence $w=u$ in $B$ by classical uniqueness results of viscosity solution of linear PDE in a bounded domain(Cf. [7]). Hence,

$$
u \in C^{2, \alpha}(B)
$$

Finally, if $D \Subset \mathcal{C}$, then $\bar{D}$ can be covered by finitely many open balls contained in $\mathcal{C}$, hence

$$
u \in C^{2, \alpha}(\bar{D})
$$

Now we are are ready to establish the main regularity theorem.

Theorem 4.2 ( $W_{\text {loc }}^{2, p}$-Regularity). Assume that

$$
\sigma \in C^{1,1}(D) \text { for any compact set } D \subset \mathbb{R}^{n} .
$$

Then for any bounded open set $\mathcal{O} \subset \mathbb{R}^{n}$, and $p<\infty$ we have

$$
u \in W^{2, p}(\mathcal{O}) .
$$

In particular, $u \in C^{1}\left(\mathbb{R}^{n}\right)$, by the Sobolev embedding.

Proof. Given any bounded open set $\mathcal{O}$, we denote by $\mathcal{C}^{\prime}\left(\mathcal{A}^{\prime}\right.$, resp.) the restriction of the continuation (action, resp.) region within $\mathcal{O}$.

Our approach is to prove, for some constant $C$ depending on $\mathcal{O}$,

$$
-C \leq \mathcal{L} u \leq C
$$

${ }^{2} D \Subset \mathcal{C}$ means that $D$ is compactly contained in $\mathcal{C}$, i.e., there exists a compact set $F$ such that $D \subset F \subset \mathcal{C}$ ). 
in the sense of distribution. That is, for any smooth test function $\varphi \in C_{c}^{\infty}(\mathcal{O})$ with $\varphi \geq 0$, we have

$$
-C \int_{\mathcal{O}} \varphi d x \leq \int_{\mathcal{O}}\left(a_{i j} u_{x_{i}} \varphi_{x_{j}}+b_{i} u_{x_{i}} \varphi+r u \varphi\right) d x \leq C \int_{\mathcal{O}} \varphi d x
$$

First, by Eq. (4.2), we can write the differential operator $\mathcal{L}$ into divergence form

$$
\mathcal{L} u=-\left(a_{i j} u_{x_{i}}\right)_{x_{j}}-b_{i} u_{x_{i}}+r u,
$$

with $a_{i j} \in C^{1,1}(\overline{\mathcal{O}})$ and $b_{i}$ Lipschitz. Note also that the first weak derivatives $u_{x_{i}}$ are welldefined since $u$ is Lipschitz $\left(u \in W^{1, \infty}(\mathcal{O})\right)$.

Observe that $u$ is a viscosity subsolution of $\mathcal{L} u=f$ in $\mathcal{O}$ since it is a viscosity solution of Eq. (HJB). Thus $u$ is also a distribution subsolution according to H. Ishii [15, Theorem 1]. Hence we have

$$
\mathcal{L} u \leq C
$$

in the sense of distribution, with $C=\sup _{\overline{\mathcal{O}}}|f|$.

Next, we show that

$$
\mathcal{L} u^{\varepsilon}\left(x_{0}\right) \geq-C, \quad \forall x_{0} \in \mathcal{O},
$$

where $u^{\varepsilon}=u * \eta^{\varepsilon} \in C^{\infty}$ is the mollification of $u, \eta^{\varepsilon}=\eta(x / \varepsilon) / \varepsilon^{n}$ and $\eta(\cdot)$ is the standard mollifier. This is proved according to three different cases.

Case 1. $x_{0} \in \mathcal{C}^{\prime}$. Then by Lemma 4.1, $\mathcal{L} u\left(x_{0}\right)=f\left(x_{0}\right)$ in the classical sense. Hence

$$
\left|\mathcal{L} u^{\varepsilon}\left(x_{0}\right)\right|=\left|f^{\varepsilon}\left(x_{0}\right)\right| \leq C,
$$

where $C=\sup _{\overline{\mathcal{O}}}|f|$, which does not depend on $x_{0}$.

Case 2. $x_{0} \in \partial \mathcal{A}^{\prime}$. Then there exists a sequence $\left\{x_{n}\right\} \subset \mathcal{C}^{\prime}$ converging to $x_{0}$. Since $\left|\mathcal{L} u^{\varepsilon}\left(x_{n}\right)\right| \leq C$ for all $n$ by the proof in Case 1, we obtain (4.6) by taking the limit as $n \rightarrow \infty$.

Case 3. $x_{0} \in \operatorname{Int} \mathcal{A}^{\prime}$, the interior of $\mathcal{A}^{\prime}$. Since $\mathcal{A}^{\prime} \subset \mathcal{O}$ is bounded, and $|B(\xi)| \rightarrow \infty$ as $|\xi| \rightarrow \infty$, we can find an open ball $\mathcal{O}^{\prime} \supset \mathcal{O}$ so that $\xi(y) \in \mathcal{O}^{\prime}$ for all $y \in \mathcal{A}^{\prime}$ and $\xi(y) \in \Xi(y)$, because

$$
B(\xi(y))=\mathcal{M} u(y)-u(y+\xi(y)) \leq \mathcal{M} u(y) \leq \sup _{\overline{\mathcal{O}^{\prime}}} \mathcal{M} u
$$

Now we define the set

$$
D:=\left\{y \in \mathcal{O}^{\prime}: u(y)<\mathcal{M} u(y)-\frac{K}{2}\right\} .
$$

Clearly, $D \Subset \mathcal{C}$. From Lemma 4.1 ,

$$
u \in C^{2, \alpha}(\bar{D})
$$

For any $x \in \operatorname{Int} \mathcal{A}^{\prime}$, suppose $B_{\rho_{1}}(x) \subset \operatorname{Int} \mathcal{A}^{\prime}$. Let us take $\xi(x) \in \Xi(x)$, then $y:=x+\xi(x)$ satisfies

$$
u(y)-\mathcal{M} u(y) \leq K
$$


by Proposition 2, Hence $y \in D$.

On the other hand, since $u-\mathcal{M} u$ is uniformly continuous on $\overline{\mathcal{O}^{\prime}}$, there exists $\rho_{2}>0$ such that

$$
\left|y-y^{\prime}\right| \leq \rho_{2} \Rightarrow\left|u\left(y^{\prime}\right)-\mathcal{M} u\left(y^{\prime}\right)-(u(y)-\mathcal{M} u(y))\right| \leq \frac{K}{4} .
$$

Therefore, for any constant $\lambda \in[-1,1]$ and any unit vector $\chi \in \mathbb{R}^{n}, y^{\prime}=y+\lambda \rho_{2} \chi$ satisfies

$$
u\left(y^{\prime}\right)-\mathcal{M} u\left(y^{\prime}\right) \leq u(y)-\mathcal{M} u(y)+\frac{K}{4}<-\frac{K}{2} .
$$

Hence, if we let $0<\rho \leq \rho_{1} \wedge \rho_{2}$, then for all $\lambda \in[-1,1], \chi \in \mathbb{R}^{n}$, with $|\chi|=1$, we have

$$
\begin{aligned}
& y=x+\xi(x) \in D, \quad y^{\prime}=y+\lambda \rho \chi \in D, \\
& x \in \operatorname{Int} \mathcal{A}^{\prime}, \quad x+\lambda \rho \chi \in \operatorname{Int} \mathcal{A}^{\prime} .
\end{aligned}
$$

By definition, we obtain,

$$
\begin{gathered}
u(x)=\mathcal{M} u(x)=u(x+\xi(x))+B(\xi(x)), \\
u(x \pm \rho \chi)=\mathcal{M} u(x \pm \rho \chi) \leq u(x \pm \rho \chi+\xi(x))+B(\xi(x)),
\end{gathered}
$$

and hence the second difference quotient at $x$

$$
\begin{aligned}
& \frac{1}{\rho^{2}}[u(x+\rho \chi)+u(x-\rho \chi)-2 u(x)] \\
\leq & \frac{1}{\rho^{2}}[u(y+\rho \chi)+u(y-\rho \chi)-2 u(y)] \\
= & \frac{1}{|\rho|} \int_{0}^{1}[(D u(y+\lambda \rho \chi)-D u(y-\lambda \rho \chi)] \cdot \chi d \lambda \\
\leq & C_{D},
\end{aligned}
$$

where $C_{D}=\sup _{x \in \bar{D}}\left|D^{2} u(x)\right| \leq\|u\|_{C^{2, \alpha}(\bar{D})}$.

Now, $x_{0} \in \operatorname{Int} \mathcal{A}^{\prime}$ given, suppose $B_{\theta}\left(x_{0}\right) \subset \operatorname{Int} \mathcal{A}^{\prime}$, then for any $0<\varepsilon<\frac{\theta}{2}, \rho_{1}=\frac{\theta}{2}, z \in$ $B_{\varepsilon}(0)$, we have $B_{\rho_{1}}\left(x_{0}-z\right) \subset \operatorname{Int} \mathcal{A}^{\prime}$. Therefore, for $0<\rho \leq \rho_{1} \wedge \rho_{2}$, and unit vector $\chi \in \mathbb{R}^{n}$,

$$
\begin{aligned}
& \frac{1}{\rho^{2}}\left[u^{\varepsilon}\left(x_{0}+\rho \chi\right)+u^{\varepsilon}\left(x_{0}-\rho \chi\right)-2 u^{\varepsilon}\left(x_{0}\right)\right] \\
= & \frac{1}{\rho^{2}} \int_{B_{\varepsilon}(0)}\left[u\left(x_{0}-z+\rho \chi\right)+u\left(x_{0}-z-\rho \chi\right)-2 u\left(x_{0}-z\right)\right] \eta^{\varepsilon}(z) d z \\
\leq & C_{D} \int_{B_{\varepsilon}(0)} \eta^{\varepsilon}(z) d z=C_{D} .
\end{aligned}
$$

Sending $\rho \rightarrow 0$ we get

$$
\chi^{\top} D^{2} u^{\varepsilon}\left(x_{0}\right) \chi \leq C_{D}
$$


Hence,

$$
\begin{aligned}
\operatorname{tr}\left(\sigma\left(x_{0}\right) \sigma\left(x_{0}\right)^{\top} D^{2} u^{\varepsilon}\left(x_{0}\right)\right) & =\operatorname{tr}\left(\sigma^{\top}\left(x_{0}\right) D^{2} u^{\varepsilon}\left(x_{0}\right) \sigma\left(x_{0}\right)\right) \\
& =\sum_{k} \sigma_{k}^{\top} D^{2} u^{\varepsilon} \sigma_{k} \\
& \leq C_{D} \sum_{i, j}\left|\sigma_{i j}\left(x_{0}\right)\right|^{2} \\
& \leq C,
\end{aligned}
$$

where $\sigma_{k}$ is the $k$-th column of the matrix $\sigma, \sigma_{i j}$ is the $(i, j)$-th element of $\sigma$, and the last inequality is due to continuity of $\sigma$.

Note that $\left|u^{\varepsilon}\left(x_{0}\right)\right|+\left|D u^{\varepsilon}\left(x_{0}\right)\right| \leq\|u\|_{W^{1, \infty}(\overline{\mathcal{O}})}$ and $\mu(x)$ bounded, we deduce

$$
\mathcal{L} u^{\varepsilon}\left(x_{0}\right)=-\frac{1}{2} \operatorname{tr}\left(\sigma\left(x_{0}\right) \sigma\left(x_{0}\right)^{\top} D^{2} u^{\varepsilon}\left(x_{0}\right)\right)-\mu\left(x_{0}\right) \cdot D u^{\varepsilon}\left(x_{0}\right)+r u^{\varepsilon}\left(x_{0}\right) \geq-C,
$$

where $C$ is independent of $x_{0}$.

Finally, (4.6) implies that for any smooth test function $\varphi \in C_{c}^{\infty}(\mathcal{O}), \varphi \geq 0$,

$$
\int_{\mathcal{O}}\left(a_{i j} u_{x_{i}}^{\varepsilon} \varphi_{x_{j}}+b_{i} u_{x_{i}}^{\varepsilon} \varphi+r u^{\varepsilon} \varphi\right) d x \geq-C \int_{\mathcal{O}} \varphi d x .
$$

Since $u \in W^{1,2}(\mathcal{O}), u^{\varepsilon} \rightarrow u$ in $L^{2}(\mathcal{O})$, and $u_{x_{i}}^{\varepsilon} \rightarrow u_{x_{i}}$ in $L^{2}(\mathcal{O})$. Sending $\varepsilon \rightarrow 0$ in the above inequality, we obtain (4.3). Therefore,

$$
\mathcal{L} u \in L^{\infty}(\mathcal{O}) .
$$

By Caldron-Zygmund estimate (see, e.g. [11]),

$$
u \in W^{2, p}(\mathcal{O}), \quad \forall p<\infty .
$$

Remark 2. Compared to the regularity results in [1], we deal with a control which is unbounded and not necessarily positive. Moreover, we prove the regularity property for the value function as a viscosity solution of HJB, as opposed to their weak solutions of QVI with $u \in H_{0}^{1}$ satisfying

$$
\left\{\begin{array}{l}
a(u, v-u) \geq\langle f, v-u\rangle, \quad \forall v \in H_{0}^{1}, v \leq \mathcal{M} u \\
0 \leq u \leq \mathcal{M} u
\end{array}\right.
$$

where $a(\phi, \psi)=\langle\mathcal{L} \phi, \psi\rangle$ and $\langle\cdot, \cdot\rangle$ is the paring between the Hilbert space $H_{0}^{1}$ and its dual space. (See Lemma 2.3-2.4 and Theorem 2.2 in Chapter 4). In addition, their key lemma, Lemma 2.3, requires, in our notation, $\gamma(x):=i n f_{x+\xi \in \partial O} B(\xi) \in W^{2, \infty}(O)$ and therefore $C^{1}$. However, this condition fails in our case, see our example in Section 5 where the corresponding $\gamma$ has a corner. 


\section{Structure of the Value Function}

Having obtained the regularity results for the value function, in this section we shall characterize the structure of the value function as well as the continuation/action regions for the following special case: $n=1$ and the cost functions $f$ and $B$ are given by

$$
\begin{aligned}
f(x) & = \begin{cases}h x, & \text { if } x \geq 0 \\
-p x, & \text { if } x \leq 0\end{cases} \\
B(\xi) & = \begin{cases}K^{+}+k^{+} \xi, & \text { if } \xi \geq 0, \\
K^{-}-k^{-} \xi, & \text { if } \xi<0,\end{cases}
\end{aligned}
$$

where $h, p, k^{+}, k^{-}, K^{+}, K^{-}$are positive constants. Moreover, we assume that $\mu$ and $\sigma$ are all constant:

$$
\sigma(x) \equiv \sigma, \quad \mu(x) \equiv \mu .
$$

In addition, we will impose the following condition to rule out triviality,

$$
h-r k^{-}>0, \quad p-r k^{+}>0 .
$$

This case was first characterized in [6] with a verification type argument. Here we provide an alternative derivation by exploiting the regularity property established earlier. We shall show directly

Theorem 5.1 (Characterization of the Solution Structure). Assuming (5.1), (5.2), (5.3), and (5.4).

(1) There exist constants $-\infty<q<s<\infty$ such that

$$
\begin{aligned}
& \mathcal{C}:=\{x \in \mathbb{R}: u(x)<\mathcal{M} u(x)\}=(q, s), \\
& \mathcal{A}:=\{x \in \mathbb{R}: u(x)=\mathcal{M} u(x)\}=(-\infty, q] \cup[s,+\infty) .
\end{aligned}
$$

(2) The value function $u$ defined in (2.3) satisfies

$$
\begin{cases}\mathcal{L} u(x)=f(x), & q<x<s \\ u(x)=u(s)+k^{-}(x-s), & x \geq s \\ u(x)=u(q)+(q-x) k^{+}, & x \leq q .\end{cases}
$$

(3) There are points $Q, S \in(q, s)$ such that

$$
\begin{array}{ll}
u^{\prime}(q)=u^{\prime}(Q)=-k^{+}, & u(q)=u(Q)+K^{+}+k^{+}(Q-q), \\
u^{\prime}(s)=u^{\prime}(S)=k^{-}, & u(s)=u(S)+K^{-}-k^{-}(S-s),
\end{array}
$$

as shown in Figure 1. 


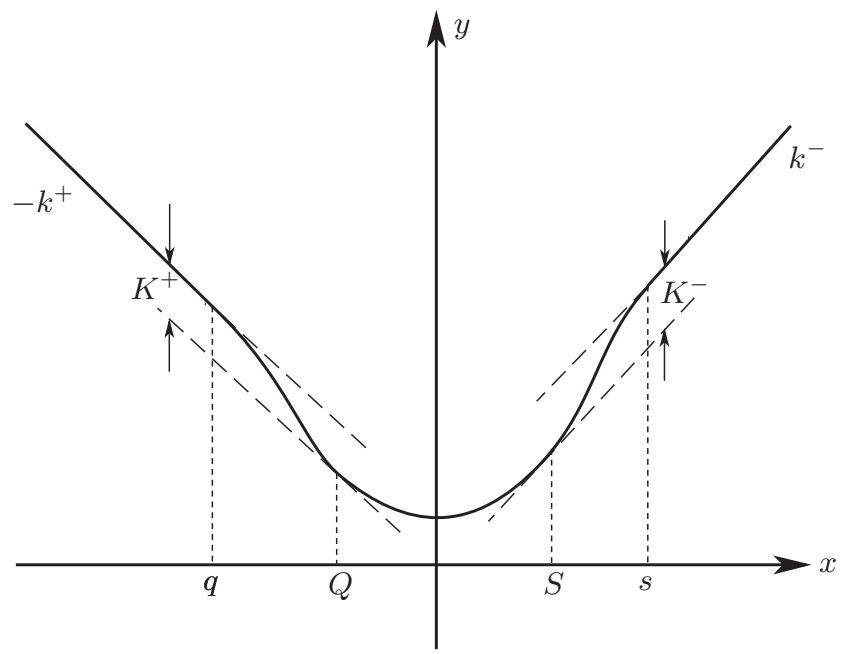

Figure 1: The Value Function $u$

The proof of Theorem 5.1 is based on a series of lemmas.

Lemma 5.2. Under assumptions (5.1) and (5.2), for any $x_{0} \in \mathcal{A}$ and

$$
\xi_{0} \in \Xi\left(x_{0}\right):=\left\{\xi \in \mathbb{R}: \mathcal{M} u\left(x_{0}\right)=u\left(x_{0}+\xi\right)+B(\xi)\right\},
$$

we have

$$
u^{\prime}\left(x_{0}\right)=u^{\prime}\left(x_{0}+\xi_{0}\right)= \begin{cases}-k^{+}, & \xi_{0}>0, \\ k^{-}, & \xi_{0}<0 .\end{cases}
$$

Proof. First, such a $\xi_{0}$ exists and $\xi_{0} \neq 0$ by Proposition 2. By definition, $u\left(x_{0}\right)=\mathcal{M} u\left(x_{0}\right)=$ $u\left(x_{0}+\xi_{0}\right)+B\left(\xi_{0}\right)$, which means $\xi_{0}$ is a global minimum of the function $u\left(x_{0}+\cdot\right)+B(\cdot)$. And $\xi_{0} \neq 0$ implies that $B$ is also differentiable at $\xi_{0}$, hence

$$
u^{\prime}\left(x_{0}+\xi_{0}\right)=-B^{\prime}\left(\xi_{0}\right)= \begin{cases}-k^{+}, & \xi_{0}>0, \\ k^{-}, & \xi_{0}<0 .\end{cases}
$$

Now, for any $\delta \neq 0$, we have

$$
u\left(x_{0}+\delta\right) \leq \mathcal{M} u\left(x_{0}+\delta\right) \leq u\left(x_{0}+\delta+\xi_{0}\right)+B\left(\xi_{0}\right) .
$$

Thus,

$$
\begin{array}{ll}
\frac{u\left(x_{0}+\delta\right)-u\left(x_{0}\right)}{\delta} \leq \frac{u\left(x_{0}+\xi_{0}+\delta\right)-u\left(x_{0}+\xi_{0}\right)}{\delta}, & \delta>0, \\
\frac{u\left(x_{0}+\delta\right)-u\left(x_{0}\right)}{\delta} \geq \frac{u\left(x_{0}+\xi_{0}+\delta\right)-u\left(x_{0}+\xi_{0}\right)}{\delta}, & \delta<0 .
\end{array}
$$

Taking the limit as $\delta \rightarrow 0^{+}, \delta \rightarrow 0^{-}$respectively, we conclude that

$$
u^{\prime}\left(x_{0}\right)=u^{\prime}\left(x_{0}+\xi_{0}\right) .
$$


Lemma 5.3. Assume (5.1), (5.2), and(5.3). For any $x_{0} \in \mathcal{A}$ and $\xi_{0} \in \Xi\left(x_{0}\right)$,

(1) if $x_{0}>0$, then $\xi_{0}<0$ and $u^{\prime}\left(x_{0}\right)=k^{-}$;

(2) if $x_{0}<0$, then $\xi_{0}>0$ and $u^{\prime}\left(x_{0}\right)=-k^{+}$.

Proof. (1) Suppose not, then there exists a $\xi_{0} \in \Xi\left(x_{0}\right)$ with $\xi_{0}>0$ and

$$
u\left(x_{0}\right)=\mathcal{M} u\left(x_{0}\right)=u\left(x_{0}+\xi_{0}\right)+B\left(\xi_{0}\right) .
$$

First, take an $\varepsilon$-optimal strategy $V=\left(\tau_{1}, \xi_{1} ; \tau_{2}, \xi_{2} ; \ldots\right)$ for the initial level $x_{0}+\xi_{0}$, i.e.,

$$
J_{x_{0}+\xi_{0}}[V] \leq u\left(x_{0}+\xi_{0}\right)+\varepsilon,
$$

where $\varepsilon>0$ arbitrarily small, to be chosen later.

Construct a strategy for $x_{0}$,

$$
V_{1}=\left(0, \xi_{0} ; \tau_{1}, \xi_{1} ; \tau_{2}, \xi_{2} ; \ldots\right)
$$

Then by definition,

$$
J_{x_{0}}\left[V_{1}\right]=J_{x_{0}+\xi_{0}}[V]+B\left(\xi_{0}\right) \leq u\left(x_{0}+\xi_{0}\right)+\varepsilon+B\left(\xi_{0}\right)=u\left(x_{0}\right)+\varepsilon .
$$

On the other hand, we can construct another strategy for $x_{0}$,

$$
V_{2}=\left(\tau, \xi_{0} ; \tau_{1}, \xi_{1} ; \tau_{2}, \xi_{2} ; \ldots\right)
$$

where

$$
\tau=\inf \left\{t: x\left(t ; x_{0}, V\right)<0\right\} .
$$

Here, we use $x\left(t ; x_{0}, V\right)$ to denote the solution of (2.1) with initial value $x_{0}$ and strategy $V$.

Since the system is linear by (5.3), we have

$$
x\left(t ; x_{0}, V_{2}\right)= \begin{cases}x\left(t ; x_{0}, V_{1}\right)-\xi_{0}>0, & t<\tau ; \\ x\left(t ; x_{0}, V_{1}\right), & t \geq \tau .\end{cases}
$$

It follows that $f\left(x\left(t ; x_{0}, V_{2}\right)\right) \leq f\left(x\left(t ; x_{0}, V_{1}\right)\right)$ for all $t$. So

$$
\begin{aligned}
& J_{x_{0}}\left[V_{2}\right]-J_{x_{0}}\left[V_{1}\right] \\
= & \mathbb{E}\left(\int_{0}^{\infty}\left[f\left(x\left(t ; x_{0}, V_{2}\right)\right)-f\left(x\left(t ; x_{0}, V_{1}\right)\right)\right] e^{-r t} d t+e^{-r \tau} B\left(\xi_{0}\right)-B\left(\xi_{0}\right)\right) \\
\leq & B\left(\xi_{0}\right)\left(\mathbb{E} e^{-r \tau}-1\right)=:-\nu .
\end{aligned}
$$

It remains to show that $\nu>0$.

Claim: $\tau>0$ a.s. if $\varepsilon$ is sufficiently small.

Clearly, $\tau \geq \tau_{1} \wedge \inf \left\{t: x_{0}+\mu t+\sigma W(t)<0\right\}$ and obviously $\inf \left\{t: x_{0}+\mu t+\sigma W(t)<\right.$ $0\}>0$, a.s., since $x_{0}>0$. Now we need to prove $\tau>0$ a.s. Suppose not, then

$$
\begin{aligned}
J_{x_{0}+\xi_{0}}[V] & =\mathbb{E}\left\{J_{x_{0}+\xi_{0}+\xi_{1}}\left[V \backslash\left(\tau_{1}, \xi_{1}\right)\right]+B\left(\xi_{1}\right)\right\} \\
& \geq \mathbb{E}\left\{u\left(x_{0}+\xi_{0}+\xi_{1}\right)+B\left(\xi_{1}\right)\right\} \\
& \geq \mathcal{M} u\left(x_{0}+\xi_{0}\right) .
\end{aligned}
$$


However, since $x_{0}+\xi_{0} \in \mathcal{C}$ by Proposition 2, if we take $0<\varepsilon \leq\left(\mathcal{M} u\left(x_{0}+\xi_{0}\right)-u\left(x_{0}+\xi_{0}\right)\right) / 2$, we have

$$
J_{x_{0}+\xi_{0}}[V] \leq u\left(x_{0}+\xi_{0}\right)+\varepsilon=\mathcal{M} u\left(x_{0}+\xi_{0}\right)-\varepsilon .
$$

Contradiction. Thus we proved the claim, and it follows that, $\nu=-B\left(\xi_{0}\right)\left(\mathbb{E} e^{-r \tau}-1\right)>0$.

Combining (5.6) and (5.7) and taking $\varepsilon<\nu / 2$,

$$
J_{x_{0}}\left[V_{2}\right] \leq J_{x_{0}}\left[V_{1}\right]-\nu \leq u\left(x_{0}\right)+\varepsilon-\nu<u\left(x_{0}\right)-\nu / 2,
$$

. This is a contradiction and we have $\xi_{0}>0$. It follows from Lemma 5.2 that $u^{\prime}\left(x_{0}\right)=k^{-}$.

The proof of (2) is exactly the same.

Recall $\mathcal{C}$ is an open set, i.e., a union of open intervals. The following lemma rules out the possibility that $\mathcal{C}$ contains unbounded intervals.

Lemma 5.4. Under assumption (5.4), $\mathcal{C}$ does not contain any of the intervals $(a,+\infty)$ or $(-\infty, b)$, with $a \geq-\infty, b \leq+\infty$.

Proof. Suppose $\mathcal{C} \supset(a,+\infty)$. Then we have, for $c>\max \{a, 0\}$,

$$
-\frac{1}{2} \sigma^{2} u^{\prime \prime}-\mu u^{\prime}+r u=h x, \quad x \in(c,+\infty) .
$$

The ODE has a general solution

$$
u(x)=C_{1} e^{\lambda_{1} x}+C_{2} e^{\lambda_{2} x}+\frac{h x}{r}+\frac{\mu h}{r^{2}},
$$

where $\lambda_{1}=\frac{-\mu-\sqrt{\mu^{2}+2 \sigma^{2} r}}{\sigma^{2}}<0, \quad \lambda_{2}=\frac{-\mu+\sqrt{\mu^{2}+2 \sigma^{2} r}}{\sigma^{2}}>0 . \quad$ Note that $C_{2}=0$, otherwise $u^{\prime}(x)=C_{1} \lambda_{1} e^{\lambda_{1} x}+C_{2} \lambda_{2} e^{\lambda_{2} x}+\frac{h}{r}$ is unbounded, approaching $+\infty$ or $-\infty$ as $x \rightarrow+\infty$, which contradicts the fact that $u$ is Lipschitz.

Now, for any $x>c>0$,

$$
C_{1} e^{\lambda_{1} x}+\frac{h x}{r}+\frac{\mu h}{r^{2}}=u(x)<\mathcal{M} u(x) \leq u(c)+K^{-}-k^{-}(c-x),
$$

or

$$
\left(\frac{h}{r}-k^{-}\right) x+C_{1} e^{\lambda_{1} x}<u(c)+K^{-}-k^{-} c-\frac{\mu h}{r^{2}} .
$$

As $x \rightarrow+\infty$, we get a contradiction noticing that $h-r k^{-}>0$.

$p-r k^{+}>0$ will ensure that $\mathcal{C}$ can not contain intervals of $(-\infty, b)$ type. Therefore, we prove the lemma.

Finally, we see

Lemma 5.5. Assuming (5.1), (5.2), (5.3), and (5.4). Then $\mathcal{C}$ is connected. 

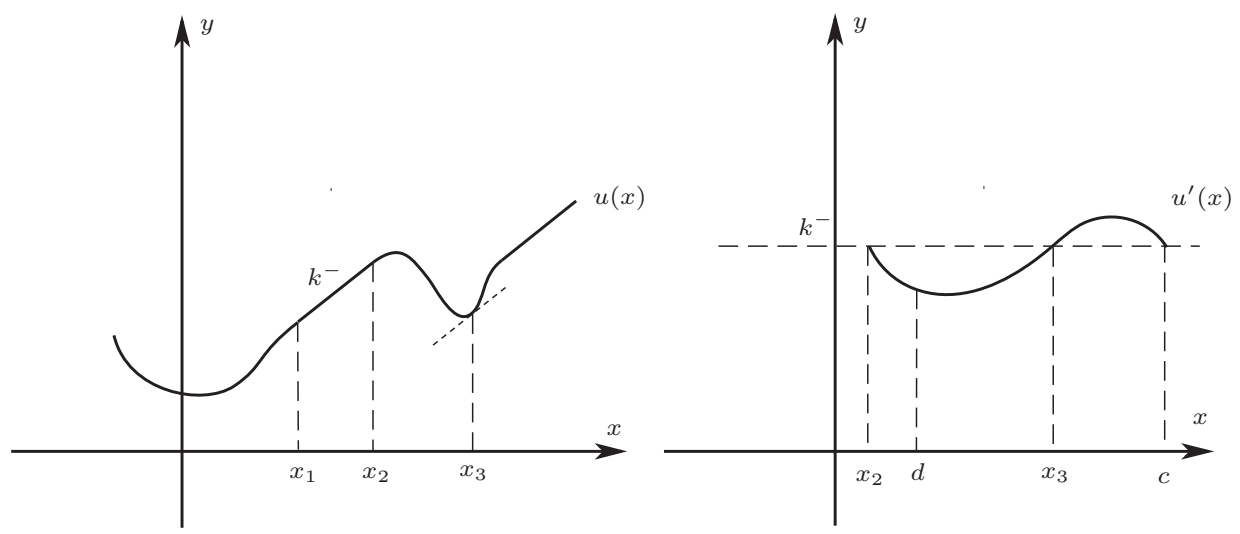

Figure 2: Proof of Theorem 5.1

Proof. Suppose not, we prove by contradiction through the following steps.

Step 1. By assumption, there are points $y_{1}<y_{2}<y_{3}$ so that $y_{1}, y_{3} \in \mathcal{C}$ while $y_{2} \in \mathcal{A}$. Define

$$
\begin{aligned}
& x_{1}:=\inf \left\{x \in \mathcal{A}: x \leq y_{2},\left[x, y_{2}\right] \subset \mathcal{A}\right\} \\
& x_{2}:=\sup \left\{x \in \mathcal{A}: x \geq y_{2},\left[y_{2}, x\right] \subset \mathcal{A}\right\} .
\end{aligned}
$$

Clearly, $x_{1}, x_{2}$ exist and are finite, with $\left[x_{1}, x_{2}\right] \subset \mathcal{A}$. (We do not rule out the possibility that $x_{1}=x_{2}$ here.)

By Lemma 5.2, $u^{\prime}(x)=k^{-}$or $-k^{+}$, for any $x \in \mathcal{A}$. Since $u \in C^{1}(\mathbb{R}), u^{\prime}$ is a constant on $\left[x_{1}, x_{2}\right]$. Assume $u^{\prime}(x)=k^{-}$, for all $x \in\left[x_{1}, x_{2}\right]$, and consider $u$ at the point $x_{2}$. (The other case $u^{\prime}=-k^{+}$is similar. In that case we consider the point $x_{1}$ instead.)

Step 2. We show that

$$
u(x) \leq u\left(x_{2}\right)+k^{-}\left(x-x_{2}\right), \quad \forall x \geq x_{2},
$$

and the inequality is strict if $x>x_{2}$ and $x \in \mathcal{C}$.

Let $\xi_{2} \in \Xi\left(x_{2}\right)$, then $\xi_{2}<0$ by Lemma 5.3 , and hence $B\left(\xi_{2}\right)=K^{-}-k^{-} \xi_{2}=B\left(\xi_{2}-y\right)-$ $k^{-} y$ for $y=x-x_{2} \geq 0$. Therefore,

$$
\begin{aligned}
& u(x) \leq(\text { or }<\text { if } x \in \mathcal{C}) \mathcal{M} u(x) \leq u\left(x_{2}+\xi_{2}\right)+B\left(x_{2}+\xi_{2}-x\right) \\
= & u\left(x_{2}+\xi_{2}\right)+B\left(\xi_{2}\right)+k^{-}\left(x-x_{2}\right)=u\left(x_{2}\right)+k^{-}\left(x-x_{2}\right) .
\end{aligned}
$$

Step 3. We show that

$$
-\mu k^{-}+r u\left(x_{2}\right) \leq h x_{2} .
$$

Since $x_{2} \in \mathcal{A}, u^{\prime}\left(x_{2}\right)=k^{-}$, Lemma 5.3 implies that $x_{2} \geq 0$. However, (5.8) implies that $x_{2}$ is a local maximum of $u-\phi$, where $\phi(x)=u\left(x_{2}\right)+k^{-}\left(x-x_{2}\right)$ is linear. By the viscosity subsolution property, we have $\mathcal{L} \phi\left(x_{2}\right) \leq f\left(x_{2}\right)=h x_{2}$, which is (5.9). 
Step 4. There exists a point $x_{3}>x_{2}$ such that

$$
-\mu k^{-}+r u\left(x_{3}\right) \geq h x_{3} .
$$

Suppose $\left(x_{2}, c\right)$ is an open interval component of $\mathcal{C}$, then by Lemma 5.4, $c<\infty$ and thus $c \in \mathcal{A}$. Lemma 5.3 implies that $u^{\prime}(c)=k^{-}$since $c>x_{2} \geq 0$. Take $d \in\left(x_{2}, c\right)$ such that $u^{\prime}(d)<k^{-}$. Such a $d$ exists since $u(x)<u\left(x_{2}\right)+k^{-}\left(x-x_{2}\right)$ for $x \in\left(x_{2}, c\right)$. Let

$$
x_{3}=\inf \left\{d \leq x \leq c: u^{\prime}(x)=k^{-}\right\} \text {, }
$$

which is well-defined, since $c$ is in this set. Clearly $x_{3}>d>x_{2}$. Moreover, $u^{\prime}(x)<k^{-}=$ $u^{\prime}\left(x_{3}\right)$ for $d \leq x<x_{3}$ by definition. So

$$
u^{\prime \prime}\left(x_{3}\right) \geq 0 .
$$

Thus $h x_{3}=-\frac{1}{2} \sigma^{2} u^{\prime \prime}\left(x_{3}\right)-\mu u^{\prime}\left(x_{3}\right)+r u\left(x_{3}\right) \leq-\mu k^{-}+r u\left(x_{3}\right)$.

Step 5. From (5.9) and (5.10), it follows that

$$
u\left(x_{3}\right)-u\left(x_{2}\right) \geq h / r\left(x_{3}-x_{2}\right)>k^{-}\left(x_{3}-x_{2}\right),
$$

by (5.4). This is a contradiction to (5.8).

Proof of Theorem 5.1. (1) Since $\mathcal{C}$ is connected, by Lemma 5.4, $\mathcal{C}=(q, s)$ for some $-\infty<$ $q<s<\infty$.

(2) For any $x \geq s, \xi \in \Xi(x)$, because $x+\xi \in \mathcal{C}=(q, s)$, we have $\xi<0$, whence $u^{\prime}(x)=k^{-}$ by Lemma 5.2. Thus, $u(x)=u(s)+k^{-}(x-s)$, for $x \geq s$. A similar argument shows that $u(x)=u(q)+(q-x) k^{+}$, for $x \leq q$.

(3) Let $\xi \in \Xi(s)$ and $S=s+\xi$. Then $S \in(q, s), u^{\prime}(s)=u^{\prime}(S)=k^{-}$and

$$
u(s)=\mathcal{M} u(s)=u(S)+B(S-s)=u(S)+K^{-}-k^{-}(S-s) .
$$

The remaining statement is similar.

\section{Appendix}

\section{Appendix A: Proof for Theorem 3.2}

Proof. (1)(Subsolution Property) Suppose $\varphi \in C^{2}\left(\mathbb{R}^{n}\right), u-\varphi$ has a local maximum at $x_{0}$ and $u\left(x_{0}\right)=\varphi\left(x_{0}\right)$. By Lemma 2.3, it suffices to prove

$$
\mathcal{L} \varphi\left(x_{0}\right)-f\left(x_{0}\right) \leq 0 .
$$

For any admissible control $V=\left\{\tau_{1}, \xi_{1} ; \tau_{2}, \xi_{2} ; \ldots\right\}$ and $\tau>0$, the control $V^{\prime}=\left\{\tau_{1}+\right.$ $\left.\tau, \xi_{1} ; \tau_{2}+\tau, \xi_{2} ; \ldots\right\}$ is also admissible, thus

$$
u\left(x_{0}\right) \leq J_{x_{0}}\left[V^{\prime}\right]=\mathbb{E}\left(\int_{0}^{\tau} f(X(t)) e^{-r t} d t+e^{-r \tau} J_{x(\tau)}[V]\right)
$$


which implies

$$
u\left(x_{0}\right) \leq \mathbb{E}\left(\int_{0}^{\tau} f(X(t)) e^{-r t} d t+e^{-r \tau} u(x(\tau))\right)
$$

where $X(t)$ is the solution of

$$
\left\{\begin{array}{l}
d X(t)=\mu(X(t)) d t+\sigma(X(t)) d W(t) \\
X(0)=x_{0}
\end{array}\right.
$$

Meanwhile, Dynkin's formula gives

$$
\mathbb{E}\left(e^{-r \tau} \varphi(x(\tau))\right)-\varphi\left(x_{0}\right)=-\mathbb{E}\left(\int_{0}^{\tau} e^{-r t} \mathcal{L} \varphi(X(t)) d t\right)
$$

Note that $u \leq \varphi$ near $x_{0}$ and $u\left(x_{0}\right)=\varphi\left(x_{0}\right)$, combining (5.11) and (5.13), and sending $\tau \rightarrow 0^{+}$, we have $\mathcal{L} \varphi-f \leq 0$ at $x_{0}$.

(2) (Supersolution Property) Suppose $\varphi \in C^{2}\left(\mathbb{R}^{n}\right), u-\varphi$ has a local minimum at $x_{0}$ and $u\left(x_{0}\right)=\varphi\left(x_{0}\right)$. If $u\left(x_{0}\right)=\mathcal{M} u\left(x_{0}\right)$, then (3.4) is trivially true. Thus we assume $u\left(x_{0}\right)<\mathcal{M} u\left(x_{0}\right)$. By continuity of $\mathcal{M}$, there exist constants $\delta>0, \rho>0$ such that

$$
\varphi(x) \leq u(x), u(x)-\mathcal{M} u(x)<-\delta, \text { whenever }\left|x-x_{0}\right|<\rho .
$$

Define

$$
\tau_{\rho}:=\inf \left\{t>0:\left|X(t)-x_{0}\right| \geq \rho\right\} .
$$

For any $\varepsilon>0$, choose an $\varepsilon$-optimal control $V=\left(\tau_{1}, \xi_{1} ; \tau_{2}, \xi_{2} ; \ldots\right)$, i.e.,

$$
J_{x_{0}}[V] \leq u\left(x_{0}\right)+\varepsilon .
$$

Then for any stopping time $\tau \leq \tau_{1}$ a.s.,

$$
u\left(x_{0}\right)+\varepsilon \geq J_{x_{0}}[V]=\mathbb{E}\left(\int_{0}^{\tau} f(X(t)) e^{-r t} d t+e^{-r \tau} J_{x\left(\tau^{-}\right)}\left[V^{\prime}\right]\right)
$$

where $V^{\prime}=\left(\tau_{1}-\tau, \xi_{1} ; \tau_{2}-\tau, \xi_{2} ; \ldots\right)$ is admissible.

Fix $R>0$ and let $\bar{\tau}=\tau_{\rho} \wedge R$.

Claim: $\mathbb{P}\left\{\tau_{1}<\bar{\tau}\right\} \rightarrow 0$ as $\varepsilon \rightarrow 0$.

Consider (5.15) with $\tau=\tau_{1}$. On the set $\left\{\tau_{1}<\bar{\tau}\right\}$,

$$
\begin{aligned}
& J_{x\left(\tau_{1}^{-}\right)}\left[V^{\prime}\right]=\mathbb{E}\left(J_{x\left(\tau_{1}^{-}\right)+\xi_{1}}[\tilde{V}]+B\left(\xi_{1}\right)\right) \\
\geq & \mathbb{E}\left(u\left(x\left(\tau_{1}^{-}\right)+\xi_{1}\right)+B\left(\xi_{1}\right)\right) \geq \mathcal{M} u\left(x\left(\tau_{1}^{-}\right)\right) \\
\geq & u\left(x\left(\tau_{1}^{-}\right)\right)+\delta,
\end{aligned}
$$


because of (5.14). Otherwise, we still have $J_{x\left(\tau_{1}^{-}\right)}\left[V^{\prime}\right] \geq u\left(x\left(\tau_{1}^{-}\right)\right)$. Thus,

$$
\begin{aligned}
u\left(x_{0}\right)+\varepsilon & \geq \mathbb{E}\left(\int_{0}^{\tau_{1}} f(X(t)) e^{-r t} d t+e^{-r \tau_{1}} J_{x\left(\tau_{1}^{-}\right)}\left[V^{\prime \prime}\right]\right) \\
& \geq \mathbb{E}\left(\int_{0}^{\tau_{1}} f(X(t)) e^{-r t} d t+e^{-r \tau_{1}} u\left(x\left(\tau_{1}^{-}\right)\right)\right)+e^{-r R} \delta \cdot \mathbb{P}\left\{\tau_{1}<\bar{\tau}\right\} \\
& \geq u\left(x_{0}\right)+e^{-r R} \delta \cdot \mathbb{P}\left\{\tau_{1}<\bar{\tau}\right\} .
\end{aligned}
$$

This proves the claim.

Take $\tau=\bar{\tau} \wedge \tau_{1}$ and sending $\varepsilon \rightarrow 0$ in (5.15), we get

$$
u\left(x_{0}\right) \geq \mathbb{E}\left(\int_{0}^{\bar{\tau}} f(X(t)) e^{-r t} d t+e^{-r \bar{\tau}} u\left(x\left(\bar{\tau}^{-}\right)\right)\right) .
$$

Note that $\varphi\left(x\left(\bar{\tau}^{-}\right)\right) \leq u\left(x\left(\bar{\tau}^{-}\right)\right), \varphi\left(x_{0}\right)=u\left(x_{0}\right)$, the above inequality together with Dynkin' formula (5.13) gives

$$
\mathbb{E}\left(\int_{0}^{\bar{\tau}} e^{-r t}(\mathcal{L} \varphi-f)(X(t)) d t\right) \geq 0 .
$$

Dividing by $\mathbb{E}(\bar{\tau})$ and sending $\rho \rightarrow 0$, we obtain the desired result (3.4).

\section{Appendix B: Proof of Lemma 3.5}

To prove this lemma, we first recall a well-known comparison theorem on elliptic PDEs.

Theorem 5.6 (Theorem 3.3 in [7]). Let $U$ be a bounded open subset of $\mathbb{R}^{n}$, and $F \in$ $C\left(U \times \mathbb{R} \times \mathbb{R}^{n} \times S^{n}\right)$ satisfy

1. $F(x, t, p, X) \leq F(x, s, p, Y)$ whenever $t \leq s, Y \leq X$,

2. There exists some $\gamma>0$ such that for $r \geq s$ and $(x, p, X) \in \bar{U} \times \mathbb{R}^{n} \times S^{n}$,

$$
\gamma(r-s) \leq F(x, r, p, X)-F(x, s, p, X) .
$$

3. There is a function $\omega:[0, \infty] \rightarrow[0, \infty]$ with $\omega(0+)=0$ such that

$$
F(y, r, \alpha(x-y), Y)-F(x, r, \alpha(x-y), X) \leq \omega\left(\alpha|x-y|^{2}+|x-y|\right)
$$

whenever $x, y \in U, r \in \mathbb{R}, X, Y \in S^{n}$, and

$$
-3 \alpha\left(\begin{array}{cc}
I & 0 \\
0 & I
\end{array}\right) \leq\left(\begin{array}{cc}
X & 0 \\
0 & Y
\end{array}\right) \leq 3 \alpha\left(\begin{array}{cc}
I & -I \\
-I & I
\end{array}\right)
$$

Let $u \in C(\bar{U})$ be a viscosity subsolution and $v \in C(\bar{U})$ a viscosity supersolution of $F=0$ in $U$ with $u \leq v$ on $\partial U$. Then $u \leq v$ in $\bar{U}$. 
Here $S^{n}$ is the collection of $n \times n$ real symmetric matrices equipped with the usual ordering and $I$ is the identity matrix.

Proof of Lemma 3.5. In view of the Lemmas 3.4 and 3.5, it suffices to verify that $F$, defined by (3.10), satisfies the conditions of Theorem 5.6. Clearly, $F$ is continuous, and

$$
\begin{gathered}
F(x, t, p, X) \leq F(x, s, p, Y) \text { whenever } t \leq s, Y \leq X . \\
F(x, t, p, X)-F(x, s, p, X)=r(t-s) .
\end{gathered}
$$

Finally, we are to prove that there exists a function $\omega:[0, \infty] \rightarrow[0, \infty]$ satisfying $\omega(0+)=0$ such that if $x, y \in D, t \in \mathbb{R}, X, Y$ symmetric satisfying for some $\alpha>0$,

$$
-3 \alpha\left(\begin{array}{cc}
I & 0 \\
0 & I
\end{array}\right) \leq\left(\begin{array}{cc}
X & 0 \\
0 & -Y
\end{array}\right) \leq 3 \alpha\left(\begin{array}{cc}
I & -I \\
-I & I
\end{array}\right)
$$

then

$$
F(y, t, \alpha(x-y), Y)-F(x, t, \alpha(x-y), X) \leq \omega\left(\alpha|x-y|^{2}+|x-y|\right) .
$$

It is easy to check that $G(x, p, X)$ and $-r g(x)$ satisfy (5.16), since $f, g \in U C\left(\mathbb{R}^{n}\right)$ (Cf. Example 3.6 in [7]). Hence

$$
\begin{aligned}
& F(y, t, \alpha(x-y), Y)-F(x, t, \alpha(x-y), X) \\
= & \max \{G(y, \alpha(x-y), Y),-r g(y)\}-\max \{G(x, \alpha(x-y), X),-r g(x)\} \\
\leq & \begin{cases}-r(g(y)-g(x)), & \text { if } G(y, \alpha(x-y), Y) \leq-r g(y) \\
G(y, \alpha(x-y), Y)-G(x, \alpha(x-y), X), & \text { otherwise }\end{cases} \\
\leq & \omega\left(\alpha|x-y|^{2}+|x-y|\right) .
\end{aligned}
$$

\section{Appendix C: Sobolev Embedding}

We summarize here some relevant results concerning embeddings of various Sobolev spaces (Cf. [10]).

Theorem 5.7 (General Sobolev Inequalities). Let $U$ be a bounded open subset of $\mathbb{R}^{n}$, with $C^{1}$ boundary. Assume $u \in W^{k, p}(U)$.

1. If

$$
k<\frac{n}{p}
$$

then $u \in L^{q}(U)$, where

$$
\frac{1}{q}=\frac{1}{p}-\frac{k}{n} .
$$

In addition,

$$
\|u\|_{L^{q}(U)} \leq C\|u\|_{W^{k, p}(U)} .
$$

Here the constant $C$ depends only on $k, p, n$ and $U$. 
2. If

$$
k>\frac{n}{p}
$$

then $u \in C^{k-\left[\frac{n}{p}\right]-1, \gamma}(\bar{U})$, where

$$
\gamma= \begin{cases}{\left[\frac{n}{p}\right]+1-\frac{n}{p},} & \text { if } \frac{n}{p} \text { is not an integer } \\ \text { any positive number }<1, & \text { if } \frac{n}{p} \text { is an integer. }\end{cases}
$$

In addition,

$$
\|u\|_{C^{k-\left[\frac{n}{p}\right]-1, \gamma}(\bar{U})} \leq C\|u\|_{W^{k, p}(U)},
$$

where the constant $C$ depends only on $k, p, n, \gamma$ and $U$.

\section{References}

[1] A. Bensoussan and J.-L. Lions. Impulse Contorl and Quasivariational Inequalities. Bordas, 1982. Translation of Contrôle Impulsionnel et Inéquations Quasi-variationnelles.

[2] T. Bielecki and S. Pliska. Risk sensitive asset management with fixed transaction costs. Finance and Stochastics, 4:1-33, 2000.

[3] B. Bruder and H. Pham. Impulse control problem on fimite horizon with excution delay. Preprint, Sept 2007.

[4] A. Cadenillas, T. Choulli, M. Taksar, and L. Zhang. Classical and impulse stochastic control for the optimization of the dividend and risk policies of an insurance firm. Mathematical Finance, 16(1):181-202, 2006.

[5] A. Cadenillas and F. Zapatero. Optimal central bank intervention in the foreign exchange market. Journal of Econ. Theory, 97:218-242, 1999.

[6] G. M. Constantinides and S. F. Richard. Existence of optimal simple policies for discounted-cost inventory and cash management in continuous time. Oper. Res., 26(4):620-636, 1978.

[7] M. G. Crandall, H. Ishii, and P. L. Lions. User's guide to viscosity solutions of second order partial differential equations. Bull. Amer. Math. Soc., 27:1-62, 1992. Also available online http://arxiv.org/PS_cache/math/pdf/9207/9207212v1.pdf.

[8] M. G. Crandall, R. T. Newcomb, and Y. Tomita. Existence and uniqueness for viscosity solutions of degenerate quasilinear elliptic equations in $\mathbb{R}^{n}$. Appl. Anal., 34(1):1-23, 1989.

[9] J. E. Eastham and K. J. Hastings. Optimal impulse control of portfolio. Math. Oper. Res., 13(4):588-605, 1988. 
[10] L. C. Evans. Partial Differential Equations. AMS, 1998.

[11] D. Gilbarg and N. S. Trudinger. Elliptic Partial Differential Equations of Second Order. Springer, 1998.

[12] X. Guo and P. Tomecek. Connections between singular control and optimal switching. SIAM J. on Control and Optimization, 47(1):421-443, 2008.

[13] J. M. Harrison, T. M. Sellke, and A. J. Taylor. Impulse control of brownian motion. Math. Oper. Res., 8(3):454-466, 1983.

[14] J. M. Harrison and M. I. Taksar. Instantaneous control of Brownian motion. Math. Oper. Res., 8(3):439-453, 19831983.

[15] H. Ishii. On the equivalence of two notions of weak solutions, viscosity solutions and distribution solutions. Funkcial. Ekvac., 38:101-120, 1995.

[16] M. Jeanblanc and S. Shiryayev. Optimization of the flow of dividends. Russian Math. Surveys, 50:257-277, 1995.

[17] M. Jeanblanc-Piqué. Impulse control method and exchange rate. Mathematical Finance, 3:161-177, 1993.

[18] I. Karatzas and S. Shreve. Methods of Mathematical Finance. Springer-Verlag, 1998.

[19] R. Korn. Protfolio optimization with strictly positive transaction costs and impulse control. Finance and Stochastics, 2:85-114, 1998.

[20] R. Korn. Some applications of impulse control in mathematical finance. Math. Meth. Oper. Res., 50:493-518, 1999.

[21] P. L. Lions. Viscosity solutions of fully nonlinear second-order equations and optimal stochastic control in infinite dimensions. part i: the case of bounded stochastic evolutions. Acta Mathematica, 161(1):243-278, 1988.

[22] V. LyVath, M. Mnif, and H. Pham. A model of portfolio selection under liquidity risk and price impact. Finance and Stochastics, (1):51-90, Jan 2007.

[23] D. C. Mauer and A. Triantis. Interactions of corporate financing and investment decisions: a dynamic framework. Journal of Finance, 49(4):1253-1277, 1994.

[24] R. Merton. Continuous-time Finance. Wiley-Blackwell, 1992.

[25] A. J. Morton and S. Pliska. Optimal portfolio management with fixed transaction costs. Mathematical Finance, 5:337-356, 1995.

[26] G. Mundaca and B. Øksendal. Optimal stochastic intervention control with application to the exchange rate. J. of Mathematical Economics, 29:225-243, 1998. 
[27] B. Øksendal and K. Reikvam. Viscosity solutions of optimal stopping problems. Stoch. Stoch. Rep., 62:285-301, 1997.

[28] B. Øksendal and A. Sulem. Optimal consumption and portfolio with both fixed and proportional transaction costs. SIAM J. Cont. Optim., 40:1765-1790, 2002.

[29] B. Øksendal and A. Sulem. Optimal stochastic impulse control with delayed reaction, Preprint, 2007.

[30] A. Ormeci, J. G. Dai, and J. V. Vate. Impulse control of brownian motion: the constrained average cost case, Preprint, Georgia Institute of Technology, 2006.

[31] H. Pham. On the smooth-fit property for one-dimensional optimal switching problem. Sminaire de Probabilits, XL:187-201, 2007.

[32] M. Ramaswamy and S. Dharmatti. Uniqueness of unbounded viscosity solutions for impulse control problem. J. Math. Anal. Appl., 315:686-710, 2006.

[33] A. Sulem. A solvable one-dimensional model of a diffusion inventory system. Math. Oper. Res., 11(1):125-133, 1986.

[34] M. I. Taksar. Average optimal singular control and a related stopping problem. Math. Oper. Res., 10:63-81, 19851985.

[35] A. Triantis and J. E. Hodder. Valuing flexibility as a complex option. Journal of Finance, 45(2):549-565, 1990. 